\title{
Social and Economic Dimensions of Sleep Disorders
}

Editor

ANA C. KRIEGER

\section{SLEEP MEDICINE CLINICS}

www.sleep.theclinics.com

Consulting Editor

TEOFILO LEE-CHIONG Jr

March 2017 • Volume 12 - Number 1 